\title{
How Philosophy of Nature Needs Philosophy of Chemistry
}

\author{
Jean-Pierre Llored ${ }^{1}$ \\ (Linacre College, Oxford University)
}

\section{Introduction}

In Plato's Timaeus, we can find the name of chora $(\chi \omega \dot{\omega} \rho)$, the basic meaning of which is that of the territory of a polis (city-state), enabling it to exist in a relationship of mutual fitness. Chôra is, in the first place, the countryside which surrounds the city proper (astu), and which nourishes it. Plato uses this term by analogy as an ontological image, in which chôra becomes the nurse (tithênê) of relative Being (genesis), which was born, lives and is bound to die. Differing from absolute Being (ontôs on, eidos, idea), which exists in itself and transcends space and time, genesis cannot exist without a chôra. Both are inseparable, and this relationship is ambivalent, since the chora is not only the nurse or even the mother (mêter) of genesis, but also its imprint (ekmageion). As regards relative Being, chora - in other words the milieu of a certain being - is thus both one thing (a matrix) and its contrary (an imprint); i.e. both A and non-A. Here is an aporia which Plato never surmounts. Augustin Berque has shown that this aporia comes from the fact that Plato's rationalism relied on the principle of the excluded middle, i.e. that there cannot exist a third term which would be both A and non-A; hence, he could not intellectually admit the "third and other gender" (triton allo genos), neither absolute nor relative Being, which he nevertheless attributes to

${ }_{1}$ jean-pierre.1lored@linacre.ox.ac.be

Philosophica, 47, Lisboa, 2016, pp. 93-108. 
chôra. (Berque 2009, 2012; Pradeau 1995) This idea is very different from that of topos proposed by Aristotle, according to whom "the place of a thing is the innermost motionless boundary of what contains it," (to tou periechontos peras akinêton proton, Physics IV 212a20). This topos is a place detachable from the body, with which the body cannot have any ontological link, contrary to Plato's chôra from which genesis is not detachable. For Aristotle, what defines the identity of a body lies inside its own local boundary. Modern science has gradually reshaped this notion of topos, implementing systems of coordinates in order to localize bodies in space. Current topology is about the description of the place as if it were detachable from the body.

Modernity has always considered Nature from the dichotomy subject/object standpoint, abstracting human beings from the world and detaching the object under study from the instrument-mirror simply used to reveal its 'properties.' The advent of evolution for living beings and that of quantum physics in the domain of atomic physics have strongly reduced, at least in principle but not always in current research programmes, the relevance of such a dichotomy depending on the domains at stake. But a scrutiny of chemistry, from alchemistry to nowadays, cannot but highlight the active role of what surrounds a chemical body, say its 'milieu,' ${ }^{2}$ in the very constitution of what it is for the chemists.

The starting point of this paper is the following question: Is the milieu a spectator, a place detachable from the body under study, or, by contrast, is it an active element of its constitution? We shall take the example of chemistry in order to show that the 'spectator approach,' which underpins our representation of science, and, as a consequence, our description of Nature and the way we live on Earth, must be radically changed, thus opening the door for a kind of relationships with Nature.

\section{Chemical operations, relations, and relata}

A chemical body is defined by means of the attributes that it can display, in a precise context, against other bodies, and also by means of the operations involved to individuate it. Let us just illustrate this point using Peirce's definition of lithium:

2 We will use the notion of "associated milieu," coined by the French philosopher of Technology, Gilbert Simondon (1964), in order to insist on the fact that a "milieu" should not be understood as something already given, but rather as that complement of the individual that is brought into being by processes of individuation. In our case, the individual in progress is not a living body, or a technical object, but a chemical body. 
If you look into a textbook of chemistry for a definition of lithium, you may be told that it is that element whose atomic weight is 7 very nearly. But if the author has a more logical mind he will tell you that if you search among minerals that are vitreous, translucent, gray or white, very hard, brittle, and insoluble, for one which imparts a crimson tinge to an unluminous flame, this mineral being triturated with lime or witherite rats-bane, and then fused, can be partly dissolved in muriatic acid; and if this solution be evaporated, and the residue be extracted with sulphuric acid, and duly purified, it can be converted by ordinary methods into a chloride, which being obtained in the solid state, fused, and electrolyzed with half a dozen powerful cells, will yield a globule of a pinkish silvery metal that will float on gasolene; [then] the material of that is a specimen of lithium. (Peirce, 1931-1958, CP 2.330) ${ }^{3}$

Peirce confidently endorses the idea that lithium can be defined as a set of instructions aimed at permitting not only the identification but also the production of a specimen of lithium. This definition is clearly provisional so that the word 'lithium' will acquire new meanings as we learn more about the stuff to which it refers, using new contexts of chemical operation or new types of chemical bodies. The way a body acts depends on the way we intervene upon it. For instance, operations and instruments were essential parts of the definition of substances in eighteenth-century chemistry. Following this line of defining bodies, the French chemist and apothecary, Guillaume François Rouelle, asserted that

[c]hemistry is a physical art which, by means of certain operations and instruments, teaches us to separate the various substances which enter into the composition of bodies, and to recombine these again, either to reproduce the former bodies, or to form new ones from them (Eklund 1975, p. 2)

In Venel's description of the third column of the "Table des rapports" the relational character of chemical bodies appears to be of crucial importance. We should bear in mind that this table enabled chemists to compare the strength of the links between chemical bodies and to use them in order to predict chemical transformations and selective displacements of metals. Venel asserted that

[o]ne applies mercury to a silver dissolution in nitrous acid; this substance having more relation with this acid, than this acid has with silver,

\footnotetext{
3 Peirce's use of italics.
} 
it unites to it and precipitates silver. If one decants the liquor one will have separated silver, and on the other side mercury dissolution in nitrous acid, if one adds a lead blade to this mercury dissolution, lead has more relation with nitrous acid than mercury, it unites it and precipitates mercury. If one decants it the precipitated mercury remains on one side and on the other side a lead dissolution in nitrous acid; if one adds a copper blade to this dissolution, copper has more relation with nitrous acid and unites to it, lead will be precipitated too and there remains a copper dissolution in nitrous acid; if one adds iron copper is precipitated, if one separates as must always be done, one will have the iron dissolution. (Venel quoted and translated by Lehman 2010, p. 21)

At this period, the word chemical "operation" was used to mean what we currently call a chemical "reaction." (Holmes 1996) Notwithstanding the various change of nomenclature that occurred from this period to current chemistry and nanochemistry, instead of studying isolated bodies to be measured, compared and put into a classificatory scheme, dynamic relations between bodies have always constituted the basic set of chemical knowledge, and, at the same time, provide the grounds for the classification of the bodies themselves, as it is the case, for example, for defining scales of acidity in particular solvents. Chemical bodies-molecules and materials are defined by their selective capacity to interact with one another within a precise 'associated milieu' and a particular field of practice. In this respect, relations between bodies seem prior to substances. But "it is only because our chemical species per definition retain their identity during purification, that we are able to connect single facts of chemical relations with each other to build a systematic network of chemical knowledge." (Schummer 1998, p. 157)

Relations of transformations between bodies allow chemists to define chemical entities and properties, while operations allow them to obtain pure chemical bodies, or, more exactly, bodies having a certain degree of purity, depending on their reactivity and the chemical nature of the surroundings. Chemical purity is not an 'intrinsic' property of matter, but the temporary outcome of transformations from composites. Those purified bodies then enter into new reactions and result in new compounds which, once purified, enable chemists to widen and deepen their classification by analogy... The process is open-ended and depends on the modes of access which stabilize a certain group of relations between bodies and their surroundings.

As a consequence, if a philosopher aims to study chemistry, and maybe to think from chemistry, she/he cannot but acknowledge that within this 
domain of human activity relata cannot exist prior to relations, and that relations are not achievable without purifying operations and the presence of already purified chemical bodies. This conclusion does not stem from a logical or a linguistic study of chemical languages or reasoning only, or from an ontological perspective grounded on chemistry, but from a close inquiry about the ways chemists synthesize, purify, stabilize, and use the bodies engaged in their transformation of the world.

The first conclusion to which a close attention to chemists' activities leads is that "chemical relata and relations are constitutively co-defined within chemists' investigative and transformative enterprise: They depend on one another within an ordered and evolving network involving chemical bodies and operations." (Llored \& Bitbol 2013) As Schummer asserts "The resulting classification has turned out to be again a network structure, with substance as nodes and chemical class relation as connections." (Schummer 1998, p. 157) The way molecules and materials are obtained and defined, and the way they act upon other bodies, cannot be captured and addressed by referring either to an ontology of pure relata, or to another using relations only. Chemical grammars require relata and relations at the same time, and often use both of them in a pragmatic manner within the same discourse. At this stage of our paper, we would like to further insist on the dependence of chemical bodies and properties on the milieu within which they are present.

\section{Context-sensitiveness and mode of access dependence in chemistry}

Talking about the provisional definition of any chemical body, the historian of chemistry Ursula Klein reminds us that

[c]hemist's substances were not universal immutable objects given by nature, but things being shaped in human practice and having the same historicity and contingency as human practice. Material substances have a history. (Klein 2008, p. 41)

Emphasizing the constitutive role of operations on the definition of chemical bodies, she goes on to say that:

[t]he example of early nineteenth-century organic chemistry demonstrates that chemists' new definition and identification of organic substances was entwined with new ways of material production and individuation of these things. The nineteenth-century culture of organic 
chemistry material production and individuation, and the instruments, skills and connoisseurship involved in these activities, were as much a part of the constitution of the objects of inquiries as theories, beliefs, social interests, and power. (Klein 2008, p. 42)

In a footnote (p. 42), she even adds "I consider experimental production and individuation of objects to be part of their "constitution." This statement increasingly gains relevance as chemists explore the world, using new bodies, instruments, explanations, and models. The material production and individuation of bodies has enormously expanded in current nanochemistry, solid-state chemistry and materials science. New instrumentation and chemical devices enable chemists to explore temporal and spatial scales which have been completely unreachable until now. Chemists have gained an enlarged capacity to synthesize, scrutinize, and modify particle size and distribution, crystal structure, chemical composition, surface area, surface chemistry, surface charge, porosity, and interfaces. A 'science of individuals or particulars' arises and chemists are now able to generate and study multifarious details at the individual level. (Llored 2013) All those achievements are not solely a question of ingredients, quantities, and structure. They also depend on the devices and the instruments involved, i.e. on the milieu in which the chemical synthesis. For instance, the same ingredients, used in the same quantity, but using different acid milieu, do not bring the same chemical individual $\mathrm{ZnO}$ :

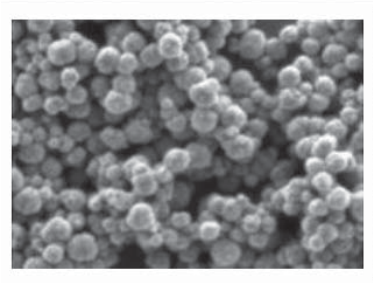

pH 5.6

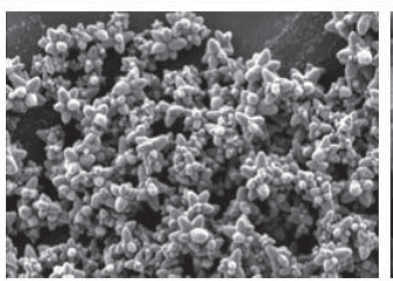

pH 11.2

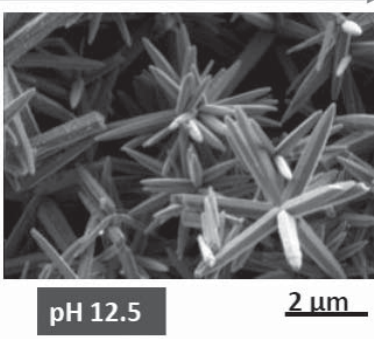

Figure 1: Polymer-assisted precipitation of $\mathrm{ZnO}$ nanoparticles with narrow particle size distribution. A. Aimable, M. T. Buscaglia, V. Buscaglia, P. Bowen, Journal of the European Ceramic Society, 30, (2010), 591-598. Anne Aimable's courtesy.

Let us take the example of the synthesis of a solid sample of $\mathrm{CaCO}_{3}$ in order to highlight the role played by the milieu both in the synthesis and the definition of a chemical body. Starting from different ingredients, 
particles will grow to attain different final sizes and morphologies. (Aimable et al. 2013) Thus, the end product may appear completely different, depending on whether a reactive material is added all at once or gradually. By adding a small amount of fine material to be precipitated (i.e. seeds), one can better control the apparently chaotic nucleation step. For example, adding calcite seeds allows for the precipitation of pure calcite. On the other hand, without seeds, one obtains a mixture of calcite and vaterite with a larger particle size distribution and various morphologies. The body $\mathrm{CaCO}_{3}$ depends on the process used and on the time employed. This body is furthermore distributed or size-dispersed in the sense that the sample does not contain a single body $\mathrm{CaCO}_{3}$ but, on the contrary, encompasses many similar bodies $\mathrm{CaCO}_{3}$ which differ in size. Neither the device nor the history of the chemical reaction can be eliminated from the final result. Operations are thus part of the definition of the 'nanobody' under study.

The mode of access cannot be eliminated from the final product insofar as it contributes to the determination of the whole body and its correlative parts and structure. The structure of the crystals may also differ if the chemical device changes. It can even differ within the same particular chemical device, depending on the size of the crystals, which itself depends on the milieu. In a nutshell, the internal arrangement can be grain-size sensitive: The concept of structure thus sometimes becomes, at least partly, extrinsic, and need further developments in such scientific technological contexts! (Llored 2013)

If a chemical body is thus tied to praxis, it is not a definite something or mere substrate that endures through time. Instead, it is at all times fully realized as just what it appears to be, and at the same time it never stays the same as it becomes transformed in processes of making, remaking, and learning to make. As an indefinite 'something out there' the chemical body enters into a series of interactions which produce determinate things that are characterized by their consistence and transformability, their performance, and their functionality. (Nordmann 2013) In the context of chemistry, the dichotomy of essences and accidents thus collapses! In The Philosophy of No, Bachelard (1940) proposed to replace the word 'substance' by that of 'ex-stance' in order to take the constitutive role of external determinations into account in the very definition of a body. Rom Harré prefers to use the term 'affordances.' (Harré 1986, 2013) An affordance can be broadly defined as a disposition or capacity as ascribed to a certain material being to yield an observable effect when acted upon in a certain manner. It may be, for instance, a gas as the product of a chemical reaction 
when certain chemical bodies are acted on during a particular electrolysis. An affordance is relative to context, in particular to the specific interaction between some human beings and the material world. What we know about chemical bodies is not about bodies in itself, even if we can determine its composition and structural characteristics, but about the ways we create, use, and transform them. Philosophizing about, and maybe from, chemistry requires changing our basic understanding of knowledge, by moving from a 'spectator theory' according to which thought is a pure representation of the world, to a 'transactional approach' to knowledge, according to which human thought is active and knowledge is about the result of our interactions with the environment.

Let us draw our second conclusion: The modes of access and the global chemical milieu in which a synthesis is carried out in chemistry do not 'reveal' pre-existing chemicals but, on the contrary, actively take part in their very constitution. Conversely, the body takes part in the redefinition and in the chemical reactivity of what surrounds it. As a result, it seems difficult to define a body at the nanoscale, or a collection of bodies at a wider scale, and the 'properties' related to them at those scales, by abstracting those bodies from other bodies or external conditions and operations required for obtaining and stabilizing them. It is not meant, of course, that it is impossible for a chemist, or a philosopher, to describe chemical bodies using their composition and their internal structure only, as if they were in isolation, and as if they were displaying intrinsic properties or dispositions only. Up to a certain point, this strategy could even turn out to be a very efficient heuristic way to produce new bodies, or to explain a certain type of reactivity during a chemical reaction. We cannot but acknowledge that such descriptions have often been used by chemists in order, for instance, to correlate the structure of a body and its chemical reactivity and properties against a biological target. But composition, that is to say, what a particular body contains, and the internal structure of a molecule or a material, can change depending on the solvent and the whole surroundings (the associated milieu), as it has been known for a long time by chemists in the case, for instance, of acid or oxidative properties.

Furthermore, as we have just shown, chemists have recently learnt that composition and internal structure can also depend on the chemical device used in nanochemistry. Knowing all the ingredients, the components of a body and their relative position from one another in space, and computing them, does not enable quantum chemists to deduce and forecast all the possible reactions and properties of a body, but only a molecular geometry, an energy threshold, or a particular kinetic or thermodynamic 
attribute of the reaction, using a host of heterogeneous models. Chemists need the chemical associated milieu as well (Llored 2012, 2014) to perform the calculation, or to carry out an experiment to identify a new property, or a type of reactivity. Structure and composition are often sufficient to practice chemistry, but not always, in particular in nanochemistry or within research projects undertaken at the frontier with material sciences where the 'milieu' of reaction becomes metastable or unstable. Sooner or later, depending on the investigation at stake, and the finer-grained description required within a particular research, the need to refer to a wider network including bodies, instruments and operations, within which the body under study gets its provisional significance and relevance, will become inescapable.

Referring to intrinsic properties, or to isolated bodies, is all but self-evident, and can even become quickly problematic in the domain of chemistry. Such 'intrinsicalness' and 'being in isolation' can play, at best, a functional role in a particular scientific or philosophical enquiry, but no more, if one takes the way chemists actually work into account. Let us now turn to our last conclusion we will draw from chemistry.

\section{Levels of organization and their interdependence in chemistry}

We have studied elsewhere the wholes/parts strategies used in many methods by quantum chemists. (Llored 2010, 2012, 2013, 2014; Harré \& Llored 2011, 2013; Banchetti-Robino \& Llored, forthcoming 2016) Whatever the differences between the different quantum chemical methods studied may be, those papers emphasize how chemists always use and intertwine the nuclei and the electrons within a molecule, the molecule itself, and the chemical milieu of the molecule containing other molecules, solvents, and electromagnetic fields, within a calculation. In the present paper, we will just give a short example, using the molecular orbital approach proposed by Robert S. Mulliken, in order to help the reader understand how strong the interdependence of the three aforementioned 'levels of organizations' are in chemists' reasoning, the reader being free to further study our other papers.

Let us consider the simple case of a molecule containing two nuclei. Our starting point is thus the molecular wave function $\psi$ which can be usefully written as a linear combination of two atomic orbitals $\varphi_{1}$ and $\varphi_{2}$ :

$$
\psi=\mathrm{c}_{1} \varphi_{1}+\mathrm{c}_{2} \varphi_{2}
$$


This equation seems to imply that the whole, which belongs to the "molecular level,' is reduced to electrons and nuclei, that is to say, to a more 'fundamental level.' Let us just examine how the weighting coefficients $\mathrm{c}_{1}$ and $\mathrm{c}_{2}$ are determined in order to understand the underlying whole/parts strategy. To do so, let us refer to Dirac's notation, where ' $\mathrm{H}$ ' is the molecular Hamiltonian: ${ }^{4}$

$$
\begin{aligned}
& <\psi_{\mathrm{i}}|\mathrm{H}| \psi_{\mathrm{i}}>=\int_{\text {space }} \psi_{i}^{*} H \psi_{i} d \tau=H_{i i} \text { is the Coulomb integral } \\
& <\psi_{\mathrm{i}}|\mathrm{H}| \psi_{\mathrm{j}}>=\int_{\text {space }} \psi_{i}^{*} H \psi_{j} d \tau=H_{i j} \text { is the exchange integral } \\
& <\psi_{\mathrm{i}} \mid \psi_{\mathrm{j}}>=\int_{\text {space }} \psi_{i}^{*} \psi_{j} d \tau=S_{i j} \text { is the overlap integral }
\end{aligned}
$$

$\varphi_{1}$ and $\varphi_{2}$ can, at least partially, overlap in the space region that corresponds to the intersection of each atomic space. The word 'orbital' takes all of its meaning from Max Born's probabilistic interpretation, according to which the square of a molecular orbital corresponds to the probability density of finding a particular electron within the molecular space. Thus,

$$
<\psi|\mathrm{H}| \psi>=c_{1}^{2}<\varphi_{1}\left|\varphi_{1}>+2 \mathrm{c}_{1} \mathrm{c}_{2} \mathrm{~S}_{12}+c_{2}^{2}<\varphi_{2}\right| \varphi_{2}>
$$

Mulliken's interpretation of the weighting coefficients $c_{i}^{2}$, where $i$ is equal to either 1 or 2 , implies that these respectively represent the part of the electron density that belongs to nucleus 1 or to nucleus 2 only. On the other hand, the term ' $2 \mathrm{c}_{1} \mathrm{c}_{2} \mathrm{~S}_{12}$ ', namely 'the overlap population,' expresses the part of the electronic density that refers to the two atomic functions at the same time and, thus, to the whole molecule: The whole and the parts are thus co-defined.

If we now consider a system (atom or molecule) in its fundamental state, and if we call $\mathrm{E}_{0}$ the system's corresponding Eigen value for the energy. The exact solution of the Schrödinger equation cannot be calculated, and chemists thus must employ a large panel of approximations. The average energy $\langle\mathrm{E}>$, calculated from an approximate wave function $\psi$, is always superior or equal to $\mathrm{E}_{0}$ :

${ }^{4} \mathrm{~d} \tau$ is a volumic element and $\psi_{i}^{*}$ the conjugate of the complex function. 


$$
\langle E\rangle=\frac{\int_{\text {space }} \psi^{*} H \psi d \tau}{\int_{\text {space }} \psi^{*} \psi d \tau} \geq E_{0}
$$

The Variation Method is currently applied in order to reach the best solution possible and enables quantum chemists to determine $c_{1}$ and $c_{2}$. The key point is to start from a family of wave functions within which chemists believe that they will find the best approximation, according to their previous calculations and to their chemical expertise. They use a wave function that depends on at least one parameter, and they determine the value of the parameter(s) (in our present case, the coefficients $c_{1}$ and $c_{2}$ ), which leads to the lowest value of the average energy. Calculations lead to the following formula:

$$
<\mathrm{E}\left(\mathrm{c}_{1}, \mathrm{c}_{2}\right)>=\frac{c_{1}^{2} H_{11}+2 c_{1} c_{2} H_{12}+c_{2}^{2} H_{22}}{c_{1}^{2}+2 c_{1} c_{2} S_{12}+c_{2}^{2}}=\frac{N\left(c_{1}, c_{2}\right)}{D\left(c_{1}, c_{2}\right)}
$$

The minimization of the energy using the coefficients $c_{1}$ and $c_{2}$ implies that:

$$
\langle E\rangle=\frac{\frac{\partial N}{\partial c_{i}}}{\frac{\partial D}{\partial c_{i}}}
$$

We simply wish to clarify this term, in order to grasp what is at stake in the calculation of those coefficients. The numerator $\mathrm{N}\left(\mathrm{c}_{1}, \mathrm{c}_{2}\right)$ is written in the following way:

$$
\mathrm{N}\left(\mathrm{c}_{1}, \mathrm{c}_{2}\right)=c_{1}^{2} H_{11}+2 c_{1} c_{2} H_{12}+c_{2}^{2} H_{22}
$$

The partial derivative calculation, using a particular coefficient while fixing the other, implies that:

$$
\frac{N\left(c_{1}, c_{2}\right)}{\partial c_{1}}=2 c_{1} H_{1}+2 c_{2} H_{\mathrm{P}} \text { and } \quad \frac{\partial N\left(c_{1}, c_{2}\right)}{\partial c_{2}}=2 c_{2} H_{2}+2 c_{1} H_{\mathrm{P}}
$$


In brief, each partial derivative depends upon: (1) the coefficients $c_{1}$ and $c_{2},(2)$ one integral $\left(\mathrm{H}_{11}\right.$ or $\left.\mathrm{H}_{22}\right)$, which refers to a unique atomic orbital $\left(\varphi_{1}\right.$ or $\varphi_{2}$, respectively), and (3) one integral $\mathrm{H}_{12}$, which deals with the energetic coupling between the two atoms inside the molecule. This situation is tantamount to saying that the two atoms intervene, particularly by means of their coupling, in the determination of each coefficient. We should bear in mind that the coupling exists once the molecule is created. In this respect, each coefficient depends upon the whole molecule and not solely upon its atoms taken in isolation! We could have drawn the same conclusion from the study of the denominator. In short, the minimization of the average energy leads to a ratio of two quantities, each depending on atoms but also on their interactions. In this respect, the weighting coefficients of the linear combination, from which one seems free to conclude that the 'molecular level' is reduced to a more 'fundamental level,' requires the whole molecule to be determined!

It is thus possible to conclude, from a technical standpoint, that there is no room for a basic level in this calculation, but only co-definitions and interrelations between different levels. In fact, if we now attempt to go beyond this technical aspect, another question inescapably arises: How can one justify the use of the Variation Principle and the minimization of energy that it implies? One needs to refer to the second principle of thermodynamics in order to explain why the molecular system continuously eliminates its excess energy by interactions with its environment. An energy transformation into local entropy legitimates the use of the variation method. The molecular whole, its parts, and the associated milieu are thus all required, at the very same time, in order to render all these methods intelligible. Chemists have contrived specific methods within which the whole and its parts are constitutively co-defined in the presence of a particular associated milieu. (Llored 2013, 2014)

\section{Concluding remarks}

Chemistry could be helpful in order to rethink Nature for at least three reasons: (1) It highlights the provisional definition of what is studied by chemists, and, above all, the context-dependence of chemical 'properties'; (2) it dissolves the representation of Nature which rests upon a basic ground from which all is reducible, simply because the way chemical operations and reasoning are construed and stabilized do not allow to draw such a conclusion; (3) it also highlights the impossibility to capture 
the concept of Nature using an ontology of relations only, as well as using an ontology of relata. Conceiving a body in isolation or, by contrast, conceiving that only relations 'exist,' is just an analytic reasoning, having a functional and heuristic interest in science and, sometimes, in philosophy.

Furthermore, chemical bodies take part in the transformation of the human and non-humans milieux, and it is this transformation that moral and political philosophers have to cope with. A milieu is a chôra, and not simply a topos. From chemistry and physics to biology and biosemiotics, from non-living to living bodies and ecosystems, we need another conceptual scheme about Nature, within which we could better integrate the mutual co-dependence between a body and its associated milieu. We are still far from accepting easily the idea that the 'reality' which surrounds us is not that of an objective environment, constituted with objects confronted by an individual subject, but is that of a 'milieu,' constituted with things which participate in our very 'Being' because of their coupling. Chemistry and its philosophy could be helpful to follow such an urgent way, and to overcome the dichotomies between the natural and the artificial or between the object and the subject.

\section{LIST OF REFERENCES}

Aimable, A., R. Brayner, J-P. Llored, M. Rozé \& S. Sarrade, "Chemistry and interfaces", in The Philosophy of Chemistry: Practices, Methodologies, and Concepts, Llored, J.-P. (ed.), Newcastle: Cambridge Scholars Publishing, 2013, pp. 172-201.

Aristotle (Written 350 B.C.E), Physics, Book IV, translated by Hardie, R. P. \& Gaye, R. K., the Internet Classics Archive: http://classics.mit.edu/Aristotle/physics.4.iv.html.

Bachelard, G., The Philosophy of No: A Philosophy of the New Scientific Mind, translation by G.C. Waterston, New York: Orion Press, 1968 [1940 in French].

Banchetti, M. \& J.-P. Llored, "Reality Without Reification: Philosophy of Chemistry's Contribution to Philosophy of Mind", in Essays in the Philosophy of Chemistry, E.R. Scerri \& G. Fisher (eds.), Oxford: Oxford University Press, 2016, pp. 77-107. 
Berque, A., "La Chôra chez Platon", in Espace et lieu dans la pensée occidentale, T. Paquot \& C. Younès, Paris: La Découverte, 2012, 13-27.

— Ecoumène. Introduction à l'étude des milieux humains, Paris: Belin, 2009.

Eklund, J., The Incompleat Chymist: Being an Essay on the Eighteenth-Century Chemist in His Laboratory, with a Dictionary of Obsolete Chemical Terms of the Period, Smithsonian Studies in History and Technology, 33, Washington, DC: Smithsonian Institute Press, 1975.

Harré, R., "Affordances and Hinges: New tools for the Philosophy of Chemistry", in The Philosophy of Chemistry: Practices, Methodologies, and Concepts, J.-P. Llored (ed.), Newcastle: Cambridge Scholars Publishing, 2013, pp. 580-96.

- Varieties of Realism. A Rational for the Natural Sciences, London: Basil Blackwell, 1986.

— \& J.-P. Llored, "Mereologies and Molecules", Foundations of Chemistry 15, issue 2 (2013), 127-44.

— \& J.-P. Llored, "Mereologies as the Grammars of Chemical Discourses", Foundations of Chemistry 13 (2011), 63-76.

Holmes, F.L., "The Communal Context for Etienne-François Geoffroy's 'Table des rapports"', Science in Context 9 (1996), 289-311.

Klein, U., “A Historical Ontology of Material Substances, C. 1700-1830”, in Stuff. The Nature of Chemical Substance, K. Ruthenberg \& J. van Brakel (eds.), Würzburg: Königshausen \& Neumann, 2008, pp. 21-44.

Lehman, C., "Innovation in Chemistry Courses in France in the Mid-Eighteenth Century: Experiments and Affinities", Ambix 57, No. 1 (2010), 3-26.

Llored, J.-P., "Wholes and Parts in Quantum Chemistry: Some Mereological and Philosophical Consequences", HYLE, International Journal for the Philosophy of Chemistry 20 (2014), 141-163.

— "Chimie, chimie quantique et concept d'émergence : étude d'une mise en relation", Ph.D. Dissertation, Free University of Brussels, November 22nd, 2013.

— \& M. Bitbol, "From Chemical Practices to a Relational Philosophy of Chemistry", in The Philosophy of Chemistry: Practices, Methodologies, and Concepts", J-P. Llored (ed.), Newcastle: Cambridge Scholars Publishing, 2013, pp. 385-415.

- "Emergence and Quantum Chemistry", Foundations of Chemistry, Volume 4, Issue 3 (2012), 245-74. 
- "Mereology and Quantum Chemistry: the Approximation of Molecular Orbital", Foundations of Chemistry, 12 (2010), 203-21.

Nordmann, A., "Metachemistry", in The Philosophy of Chemistry: Practices, Methodologies, and Concepts, J.-P. Llored, (ed.), Newcastle: Cambridge Scholars Publishing, 2013, pp. 725-43.

Peirce, C. S., The Collected Papers of C. S. Peirce, C. Hartshorne \& P. Weiss (eds.), Cambridge, Massachusetts: Harvard University Press, Volumes 1-6. The references in the text are given by volume and paragraph number.

Platon, Timée (Timaeus), Paris: Les Belles Lettres, 1925.

Pradeau, J.-F., "Etre quelque part, occuper une place", Topos et chôra dans le Timée, Les études philosophiques, 3 (1995), 375-400.

Simondon, Gilbert, L'individuation à la lumière des notions de forme et d'information. Paris: Presses universitaires de France, 1964.

Schummer, J., "The Chemical of Chemistry I: A Conceptual Approach", HYLE, International Journal for Philosophy of Chemistry, 4(2) (1998), 129-62.

\begin{abstract}
This paper aims to highlight how the philosophy of chemistry could be of help for rethinking Nature today. To do so, we will point out: (1) the co-definition of chemical relations (transformations) and chemical relata (bodies) within chemical activities; (2) the constitutive role of the modes of intervention in the definition, always open and provisional, of "active" chemical bodies; and (3) the mutual dependence of the levels of organization in chemistry. We will insist on the way chemists tailor networks of interdependencies within which chemical bodies and properties are context-sensitive and mutually determined by means of particular chemical operations or transformations. To conclude, we will show how the specific action of bodies upon the Earth at different scales of space and time, and how the relational definition of a chemical body, pave the way for a new understanding of Nature.
\end{abstract}

Keywords: philosophy of chemistry - associated milieu - levels of organization - nature - relational properties 


\section{RÉSUMÉ}

Ce texte a pour objectif d'établir comment il devient possible de repenser le concept de Nature à l'aune de la philosophie de la chimie. Pour ce faire, nous montrerons comment une épistémologie des pratiques chimiques permet de mettre en évidence : la dépendance mutuelle des niveaux d'organisation ainsi que celle des relations (transformations chimiques) et des relata (corps chimiques), et le rôle constitutif des modes d'intervention dans la définition, toujours ouverte et provisoire, de ce à quoi les chimistes disent avoir affaire. Nous insisterons sur la façon avec laquelle les chimistes définissent des réseaux d'interdépendances à l'intérieur desquels les corps chimiques et leurs propriétés dépendant des milieux dans lesquels ils se trouvent et sont définis. Pour conclure, nous montrerons comment l'action des corps chimiques sur la Terre à différentes échelles d'espace et de temps, couplée à la définition relationnelle de ces corps, ouvre de nouvelles perspectives pour repenser la Nature.

Mots Clés : philosophie de la chimie - milieu associé - niveaux d'organisation nature - propriétés relationnelles 